\title{
Borel resummation of formal solutions to nonlinear Laplace equations in 2 variables
}

\author{
by M. E. Pliś (Kraków) and B. ZiEMIAN (Warszawa)
}

\begin{abstract}
We consider a nonlinear Laplace equation $\Delta u=f(x, u)$ in two variables. Following the methods of $\mathrm{B}$. Braaksma $[\mathrm{Br}]$ and $\mathrm{J}$. Ecalle used for some nonlinear ordinary differential equations we construct first a formal power series solution and then we prove the convergence of the series in the same class as the function $f$ in $x$.
\end{abstract}

0. Introduction. We consider a nonlinear Laplace equation of the form

$$
\Delta u=\left(\frac{\partial^{2}}{\partial x_{1}^{2}}+\frac{\partial^{2}}{\partial x_{2}^{2}}\right) u=f(x, u)
$$

where $x=\left(x_{1}, x_{2}\right) \in \mathbb{R}^{2}$. First we are going to construct a formal power series solution of (1) and then prove that every such solution is of the same class as the function $f$ in $x$. Similar results for some nonlinear ordinary differential equations were proved by Braaksma $[\mathrm{Br}]$, following the ideas of J. Ecalle.

We denote by $L$ the image of the positive quadrant $\mathbb{R}_{+}^{2}=\mathbb{R}_{+} \times \mathbb{R}_{+}$under the unitary matrix $\frac{1}{\sqrt{2}}\left(\begin{array}{ccc}1-i & 1+i \\ 1+i & 1-i\end{array}\right)$.

Definition 1 ([Zie1]). A function $F$ of the variable $z=\left(z_{1}, z_{2}\right) \in \mathbb{C}^{2}$ is said to be Laplace holomorphic on $L$ if $F$ is holomorphic on some polydisk centered at $(0,0) \in \mathbb{C}^{2}$, can be holomorphically continued to some sectorial neighbourhood $S=S_{1} \times S_{2}$ of $L$ with vertex $(0,0)$, and is of exponential growth on $S$, i.e. for every closed subsector $S^{\prime}=S_{1}^{\prime} \times S_{2}^{\prime} \subset S$ there exist constants $c=\left(c_{1}, c_{2}\right)$ and $C$ such that for $z \in S^{\prime}$,

$$
\left|F\left(z_{1}, z_{2}\right)\right| \leq C e^{c_{1}\left|z_{1}\right|+c_{2}\left|z_{2}\right|} .
$$

Definition 2. A function $f$ of the variable $x=\left(x_{1}, x_{2}\right) \in \mathbb{R}^{2}$ is said to be a 1-sum of a formal power series

1991 Mathematics Subject Classification: 35C20, 44A10.

Key words and phrases: Borel resummation, formal solutions, Laplace equation, Supported by KBN grant 2-PO3A-006-08. 


$$
\widehat{f}(x)=\sum_{k, l=0}^{\infty} g_{k l} \frac{1}{\left(x_{1}+i x_{2}\right)^{k+1}\left(i x_{1}+x_{2}\right)^{l+1}}
$$

if there exists a Laplace holomorphic function $F$ on $L$ such that

$$
f(x)=\int_{L} e^{-x z} F(z) d z
$$

and

$$
F(z)=\sum_{k, l=0}^{\infty} \frac{g_{k l}}{i 2^{k+l+1} k ! l !}\left(z_{1}-i z_{2}\right)^{k}\left(z_{2}-i z_{1}\right)^{l}
$$

near zero. In that case we say that $f$ is 1-resummable.

In this paper we assume that $f(x, u)$ on the right hand side of $(1)$ is the 1 -sum in $x$ of a formal power series

$$
\widehat{f}(x, u)=\sum_{k, l=0}^{\infty} g_{k l}(u) \frac{1}{\left(x_{1}+i x_{2}\right)^{k+1}\left(i x_{1}+x_{2}\right)^{l+1}}
$$

with coefficients $g_{k l}(u)$ holomorphic for every $(k, l) \in \mathbb{N}_{0}^{2}$, on some fixed neighbourhood $U$ of zero in $\mathbb{C}$, and $g_{k l}(0)=0$.

Therefore, if we write

$$
\begin{aligned}
\widehat{f}(x, u) & =\sum_{k, l=0}^{\infty}\left(\sum_{j=1}^{\infty} c_{k l}^{j} u^{j}\right) \frac{1}{\left(x_{1}+i x_{2}\right)^{k+1}\left(i x_{1}+x_{2}\right)^{l+1}} \\
& =\sum_{j=1}^{\infty}\left(\sum_{k, l=0}^{\infty} c_{k l}^{j} \frac{1}{\left(x_{1}+i x_{2}\right)^{k+1}\left(i x_{1}+x_{2}\right)^{l+1}}\right) u^{j}
\end{aligned}
$$

then we can write $f(x, u)=\sum_{j=1}^{\infty} c_{j}(x) u^{j}$ where $c_{j}(x)$ is the 1 -sum of the formal series

$$
\sum_{k, l=0}^{\infty} c_{k, l}^{j} \frac{1}{\left(x_{1}+i x_{2}\right)^{k+1}\left(i x_{1}+x_{2}\right)^{l+1}},
$$

and $f$ is holomorphic in $u$ on $U$. Hence, we have $c_{j}(x)=\int_{L} e^{-x z} T_{j}(z) d z$ for some Laplace holomorphic functions $T_{j}$. Moreover, $T_{j}$ are holomorphic on the same sector $S$ for all $j$, and the constants $c$ and $C$ in (2) are independent of $j$.

Theorem. If $T_{j}(0) \neq 0$, then there exists a family of 1-resummable solutions of equation (1) of the form

$$
u(x)=\sum_{\nu=0}^{\infty} d_{\nu} \frac{1}{\left(x_{1}+i x_{2}\right)^{\nu_{1}+1}} \cdot \frac{1}{\left(i x_{1}+x_{2}\right)^{\nu_{2}+1}} .
$$


This means that

$$
u(x)=\int_{L} e^{-x z} T(z) d z
$$

with $T$ being a Laplace holomorphic function on L. Moreover, every formal solution $\widehat{u}$ of (1) of the above form is 1-resummable.

The proof will be divided into three parts.

1. Convolution equation. Applying $\Delta$ to $u$ in the form (3) we arrive at the complex symbol of $\Delta$ as the complex polynomial

$$
P\left(z_{1}, z_{2}\right)=z_{1}^{2}+z_{2}^{2}=\left(\frac{1+i}{\sqrt{2}} z_{1}+\frac{1-i}{\sqrt{2}} z_{2}\right)\left(\frac{1-i}{\sqrt{2}} z_{1}+\frac{1+i}{\sqrt{2}} z_{2}\right) .
$$

In the new variables

$$
\zeta_{1}=\frac{1+i}{\sqrt{2}} z_{1}+\frac{1-i}{\sqrt{2}} z_{2}, \quad \zeta_{2}=\frac{1-i}{\sqrt{2}} z_{1}+\frac{1+i}{\sqrt{2}} z_{2},
$$

$P$ becomes the polynomial $\widetilde{P}\left(\zeta_{1}, \zeta_{2}\right)=\zeta_{1} \cdot \zeta_{2}$, and after changing variables on the left hand side of (1) we get

$$
\begin{aligned}
\Delta u\left(x_{1}, x_{2}\right) & =\left(P\left(z_{1}, z_{2}\right) T\right)\left[e^{-x_{1} z_{1}-x_{2} z_{2}}\right] \\
& =\left(\widetilde{P}\left(\zeta_{1}, \zeta_{2}\right) \widetilde{T}\right)\left[e^{-x_{1}\left(\frac{1-i}{2 \sqrt{2}} \zeta_{1}+\frac{1+i}{2 \sqrt{2}} \zeta_{2}\right)-x_{2}\left(\frac{1+i}{2 \sqrt{2}} \zeta_{1}+\frac{1-i}{2 \sqrt{2}} \zeta_{2}\right)}\right] \\
& =\left(\widetilde{P}\left(\zeta_{1}, \zeta_{2}\right) \widetilde{T}\right)\left[e^{-\left(\frac{1-i}{2 \sqrt{2}} x_{1}+\frac{1+i}{2 \sqrt{2}} x_{2}\right) \zeta_{1}-\left(\frac{1+i}{2 \sqrt{2}} x_{1}+\frac{1-i}{2 \sqrt{2}} x_{2}\right) \zeta_{2}}\right] .
\end{aligned}
$$

So we are looking for a solution

$$
\widetilde{u}\left(y_{1}, y_{2}\right)=\widetilde{T}\left[e^{-y_{1} \zeta_{1}-y_{2} \zeta_{2}}\right]=u\left(\frac{1-i}{2 \sqrt{2}} x_{1}+\frac{1+i}{2 \sqrt{2}} x_{2}, \frac{1+i}{2 \sqrt{2}} x_{1}+\frac{1-i}{2 \sqrt{2}} x_{2}\right)
$$

of the convolution equation

$$
\zeta_{1} \zeta_{2} \widetilde{T}=f^{*} \widetilde{T}
$$

where $f^{*} \widetilde{T}=\sum_{j=1}^{\infty} \widetilde{T}_{j} * \widetilde{T}^{* j}$ with $\widetilde{T}^{* j}$ denoting the $j$ th convolution power of $\widetilde{T}$, i.e. $T^{* j}=T * \ldots * T$ ( $j$ times). From now on we write $T$ instead of $\widetilde{T}$.

We can assume that $T_{1}(0)=1$, for otherwise we modify slightly the change of variables after dividing equation $(1)$ by $T_{1}(0)$.

Since our existence proof for the solution of (4) essentially follows that of Braaksma [Br], we shall consider $T$ having the formal expansion

$$
T=\sum_{k, l=0}^{\infty} d_{k l} \widetilde{\zeta}_{1}^{k} \widetilde{\zeta}_{2}^{l}
$$

with $\widetilde{\zeta}^{p}=\zeta^{p} / \Gamma(p+1)$. Then due to the convolution formula

$$
\widetilde{\zeta}^{l} * \widetilde{\zeta}^{k}=\widetilde{\zeta}^{l+k+1}
$$


we find

$$
\begin{aligned}
f^{*} T & =\sum_{j=1}^{\infty} \sum_{m_{1}, m_{2}=0}^{\infty} c_{m_{1} m_{2}}^{j} \widetilde{\zeta}_{1}^{m_{1}} \widetilde{\zeta}_{2}^{m_{2}} *\left(\sum_{k, l=0}^{\infty} d_{k l} \widetilde{\zeta}_{1}^{k} \widetilde{\zeta}_{2}^{l}\right)^{* j} \\
& =\sum_{j=1}^{\infty} \sum_{m_{1}, m_{2}=0}^{\infty} c_{m_{1} m_{2}}^{j} \widetilde{\zeta}_{1}^{m_{1}} \widetilde{\zeta}_{2}^{m_{2}} * \sum_{\nu_{1}+\ldots+\nu_{j}=\mathbf{0}}^{\infty} d_{\nu_{1}} \ldots d_{\nu_{j}} \widetilde{\zeta}^{\nu_{1}+\ldots+\nu_{j}+\mathbf{j}-\mathbf{1}} \\
& =\sum_{j=1}^{\infty} \sum_{m+\nu_{1}+\ldots+\nu_{j}=0}^{\infty} c_{m}^{j} d_{\nu_{1}} \ldots d_{\nu_{j}} \widetilde{\zeta}^{m+\nu_{1}+\ldots+\nu_{j}+\mathbf{j}} \\
& =\sum_{k=\mathbf{0}}^{\infty}\left(\sum_{j=1}^{\bar{k}+1} \sum_{m+\nu_{1}+\ldots+\nu_{j}=k+\mathbf{1}-\mathbf{j}} c_{m}^{j} d_{\nu_{1}} \ldots d_{\nu_{j}}\right) \widetilde{\zeta}^{k+\mathbf{1}}
\end{aligned}
$$

for $k, m, \nu_{j} \in \mathbb{N}_{0}^{2}, \mathbf{j}=(j, j), \mathbf{1}=(1,1), \bar{k}=\min \left\{k_{1}, k_{2}\right\}$.

Inserting this in (4) we find

$$
d_{k}(k+\mathbf{1})=\sum_{j=1}^{\bar{k}+1} \sum_{m+\nu_{1}+\ldots+\nu_{j}=k+\mathbf{1}-\mathbf{j}} c_{m}^{j} d_{\nu_{1}} \ldots d_{\nu_{j}},
$$

since

$$
\zeta \cdot \widetilde{\zeta}^{p}=(p+1) \widetilde{\zeta}^{p+1} .
$$

In particular, we can take $d_{00}$ arbitrarily (since $c_{00}^{1}=1$ ), $d_{10}=c_{01}^{1} d_{00}, d_{01}$ $=c_{01}^{1} d_{00}$,

$$
\begin{aligned}
& 2 d_{20}=c_{20}^{1} d_{00}+c_{10}^{1} d_{10}, \quad 2 d_{02}=c_{02}^{1} d_{00}+c_{01}^{1} d_{01}, \\
& 3 d_{11}=c_{11}^{1} d_{00}+c_{10}^{1} d_{01}+c_{01}^{1} d_{10}+c_{00}^{2} d_{00}, \ldots
\end{aligned}
$$

We are going to prove that $T$ defined formally by (5) with coefficients $d_{\nu}$ satisfying the recurrence (6) is a holomorphic function of exponential growth in some sector $S$.

Before starting the resummation proof for the expansion (5), we consider the resummation problem with respect to one variable. Therefore, let us write (5) in the form

$$
T=\sum_{k=0}^{\infty} T_{1 k}\left(\zeta_{1}\right) \widetilde{\zeta}_{2}^{k}
$$

where $T_{1 k}\left(\zeta_{1}\right)=\sum_{l=0}^{\infty} d_{l k} \widetilde{\zeta}_{1}^{l}$.

In a way similar to that of deriving (6), we find that $T_{1 k}$ satisfy the convolution equation 


$$
\zeta_{1}(k+1) T_{1 k}=\sum_{j=1}^{k+1} \sum_{m+\nu_{1}+\ldots+\nu_{j}=k+1-j} T_{m}^{j} * T_{1 \nu_{1}} * \ldots * T_{1 \nu_{j}}
$$

for $k, m, \nu_{j} \in \mathbb{N}_{0}$, where $T_{m}^{j}=\sum_{p=0}^{\infty} c_{p m}^{j} \widetilde{\zeta}_{1}^{p}$. For $k=0$ this gives

$$
\zeta_{1} T_{10}=T_{0}^{1} * T_{10},
$$

which is equivalent to the equation

$$
\frac{d}{d t} u_{0}=c_{0}^{1}(t) u_{0}
$$

in the variable $t=\frac{1-i}{2 \sqrt{2}} x_{1}+\frac{1+i}{2 \sqrt{2}} x_{2}$.

For $k=1$ we get

$$
2 \zeta_{1} T_{11}=T_{0}^{1} * T_{11}+T_{1}^{1} * T_{10}+T_{0}^{2} * T_{10}^{* 2}
$$

or equivalently

$$
2 \frac{d}{d t} u_{1}=c_{0}^{1}(t) u_{1}+c_{1}^{1}(t) u_{0}+c_{0}^{2}(t)\left(u_{0}\right)^{2} .
$$

We easily see that the $j$ th equation is linear in $u_{j}$ with $u_{0}, \ldots, u_{j-1}$ regarded as coefficients. Since the solutions of linear equations with resummable coefficients are resummable themselves (cf. [Br], [Zie1]), we see that all $T_{1 k}$ are Laplace holomorphic functions. The same is also true for

$$
T_{l 2}\left(\zeta_{2}\right)=\sum_{j=0}^{\infty} d_{l j} \widetilde{\zeta}_{2}^{j}
$$

Now we pass to the proof of the convergence of the formal series (5) with $d_{\nu}$ satisfying (6). Since the series (5) satisfies (4), for a fixed $N \in \mathbb{N}_{0}$ the series

$$
T_{N}=\sum_{l, j=N+1}^{\infty} d_{l j} \widetilde{\zeta}_{1}^{l} \widetilde{\zeta}_{2}^{j}=T-S_{N}
$$

satisfies the equation

$$
\begin{aligned}
\zeta_{1} \zeta_{2} T_{N} & =G^{N}\left(\zeta, T_{N}\right)=\sum_{j=1}^{\infty} \sum_{k=0}^{j}\left(\begin{array}{l}
j \\
k
\end{array}\right) T_{j} * T_{N}^{* k} * S_{N}^{*(j-k)}-\zeta_{1} \zeta_{2} S_{N} \\
& =\sum_{k=0}^{\infty}\left(\sum_{\substack{j=k \\
j \geq 1}}^{\infty}\left(\begin{array}{l}
j \\
k
\end{array}\right) T_{j} * S_{N}^{*(j-k)}\right) * T_{N}^{* k}-\zeta_{1} \zeta_{2} S_{N} .
\end{aligned}
$$

We write

$$
G^{N}(\zeta, \psi)=\sum_{k=0}^{\infty} g_{k}(\zeta) * \psi^{* k}
$$


where $g_{0}=\sum_{j=1}^{\infty} T_{j} * S_{N}^{* j}-\zeta_{1} \zeta_{2} S_{N}$, and $g_{k}=\sum_{j=k}^{\infty}\left(\begin{array}{l}j \\ k\end{array}\right) T_{j} * S_{N}^{*(j-k)}$ for $k>0$. The series $g_{k}$ are convergent near $(0,0)$ due to the remarks about the resummation problem with respect to one variable and the fact that the series $\sum_{j=k}^{\infty}\left(\begin{array}{l}j \\ k\end{array}\right) T_{j}(\zeta) u^{j-k}$ is convergent near 0 . Moreover, we can see that for every subsector $S^{\prime} \subset S$ there exist $K$ and $c=\left(c_{1}, c_{2}\right)$ such that for $\zeta \in S^{\prime}$,

$$
\left\{\begin{array}{l}
\left|g_{0}(\zeta)\right| \leq K\left|\zeta_{1}\right|^{N+1}\left|\zeta_{2}\right|^{N+1} e^{c_{1}\left|\zeta_{1}\right|+c_{2}\left|\zeta_{2}\right|} \\
\left|g_{k}(\zeta)\right| \leq K e^{c_{1}\left|\zeta_{1}\right|+c_{2}\left|\zeta_{2}\right|} \quad \text { for } k \geq 1
\end{array}\right.
$$

For $p=\left(p_{1}, p_{2}\right), p_{i}>0, s=\left(s_{1}, s_{2}\right) \in \mathbb{R}^{2}$, we denote by $W_{s}(p)$ the space of functions $\psi$ holomorphic in the polydisc $\left\{\left|\zeta_{1}\right| \leq p_{1},\left|\zeta_{2}\right| \leq p_{2}\right\}$ and such that

$$
\|\psi\|_{s, p}=\sup _{\left|\zeta_{i}\right| \leq p_{i}}\left|\zeta^{-s} \psi(\zeta)\right|<\infty .
$$

Observe that for $\zeta \in\left\{\left|\zeta_{i}\right| \leq p_{i}\right\}$ and $s_{1}>-1, s_{2}>-1$,

$$
\begin{aligned}
\left|\psi^{* m}(\zeta)\right| \leq & \|\psi\|_{s, p}^{m} \frac{\Gamma\left(s_{1}+1\right)^{m} \Gamma\left(s_{2}+1\right)^{m}}{\Gamma\left(m\left(s_{1}+1\right)\right) \Gamma\left(m\left(s_{2}+1\right)\right)} \\
& \times\left|\zeta_{1}\right|^{m\left(s_{1}+1\right)-1}\left|\zeta_{2}\right|^{m\left(s_{2}+1\right)-1} .
\end{aligned}
$$

Therefore by the properties of the $\Gamma$-function the function (8) makes sense for $\psi \in W_{s}(p)$ if $s$ is large enough.

Consider the operator

$$
R \psi(\zeta)=\frac{1}{\zeta} g_{0}(\zeta)+\frac{1}{\zeta}\left(g_{1} * \psi\right)(\zeta)+\sum_{m=2}^{\infty} \frac{1}{\zeta}\left(g_{m} * \psi^{* m}\right)(\zeta)
$$

Denoting the summands by $R_{0} \psi, R_{\operatorname{lin}} \psi$ and $Q \psi$ respectively, for $\psi \in$ $W_{N-1}(p)$ and $\zeta \in\left\{\left|\zeta_{i}\right| \leq p_{i}, i=1,2\right\}$ we get the estimates

$$
\left\{\begin{aligned}
\left|R_{0} \psi(\zeta)\right| & \leq K\left|\zeta_{1}\right|^{N}\left|\zeta_{2}\right|^{N}, \\
\left|R_{\operatorname{lin}} \psi(\zeta)\right| & \leq K\|\psi\|_{N-1, p}\left|\zeta_{1}\right|^{N-1}\left|\zeta_{2}\right|^{N-1}\left(\frac{\Gamma(N)}{\Gamma(N+1)}\right)^{2} \\
& =\frac{K}{N^{2}}\|\psi\|_{N-1, p}\left|\zeta_{1} \zeta_{2}\right|^{N-1} \\
|Q \psi(\zeta)| \leq & K\left(\sum_{m=2}^{\infty}\|\psi\|_{N-1, p}^{m} \frac{\Gamma(N)^{2 m}}{\Gamma(m N)^{2}}\left|\zeta_{1} \zeta_{2}\right|^{(m-1) N-1}\right)\left|\zeta_{1} \zeta_{2}\right|^{N}
\end{aligned}\right.
$$

Set $M=\left\|\frac{1}{\zeta} g_{0}(\zeta)\right\|_{N-1, p}$. If $\|\psi\|_{N-1, p} \leq 2 M$ then by choosing $p$ small and $N$ large we may have (by (12))

$$
\|Q \psi\|_{N-1, p} \leq \frac{1}{3} M \quad \text { and } \quad\left\|R_{\operatorname{lin}} \psi\right\| \leq \frac{1}{3} M .
$$

Therefore the operator $R$ acts in the space

$$
B_{N-1, p}=\left\{\psi \in W_{N-1}(p):\|\psi\|_{N-1, p} \leq 2 M\right\} .
$$

Observe that for $\psi, \psi+\chi \in B_{N-1, p}$ we have 


$$
\begin{aligned}
\mid(\psi+\chi)^{* m}(\zeta)- & \psi^{* m}(\zeta)|=| \sum_{l=1}^{m}\left(\begin{array}{c}
m \\
l
\end{array}\right)\left(\psi^{*(m-l)} * \chi^{* l}\right)(\zeta) \mid \\
\leq & \sum_{l=1}^{m}\left(\begin{array}{c}
m \\
l
\end{array}\right)\|\psi\|_{N-1, p}^{m-l}\|\chi\|_{N-1, p}^{l} \frac{\Gamma(N)^{2 m}}{\Gamma(m N)^{2}}\left|\zeta_{1} \zeta_{2}\right|^{m N-1} \\
\leq & \frac{\Gamma(N)^{2 m}}{\Gamma(m N)^{2}}\left|\zeta_{1} \zeta_{2}\right|^{m N-1} \sum_{l=1}^{m}\left(\begin{array}{c}
m \\
l
\end{array}\right)\|\psi\|_{N-1, p}^{m-l}\|\chi\|_{N-1, p}^{l} .
\end{aligned}
$$

We have

$$
\begin{aligned}
\sum_{l=1}^{m}\left(\begin{array}{c}
m \\
l
\end{array}\right)\|\psi\|_{N-1, p}^{m-l}\|\chi\|_{N-1, p}^{l} & \leq\|\chi\|_{N-1, p} \sum_{l=1}^{m}\left(\begin{array}{c}
m \\
l
\end{array}\right)(2 M)^{m-l}(4 M)^{l-1} \\
& \leq \frac{\|\chi\|_{N-1, p}}{4 M}(2 M+4 M)^{m}=\frac{(6 M)^{m}}{4 M}\|\chi\|_{N-1, p}
\end{aligned}
$$

since $\|\chi\| \leq\|\psi+\chi\|+\|\psi\| \leq 4 M$. Hence

$$
\left|(\psi+\chi)^{* m}(\zeta)-\psi^{* m}(\zeta)\right| \leq \frac{\left(6 M \Gamma(N)^{2}\right)^{m}}{4 M \Gamma(m N)^{2}}\|\chi\|_{N-1, p}\left(\left|\zeta_{1} \zeta_{2}\right|\right)^{m N-1},
$$

and

$$
\begin{aligned}
\mid \frac{1}{\zeta}\left(g_{m} *((\psi\right. & \left.\left.+\chi)^{* m}-\psi^{* m}\right)\right)(\zeta) \mid \\
& \leq \frac{K}{4 M}\left(\frac{\left(6 M \Gamma(N)^{2}\right)^{m}}{\Gamma(m N)^{2}}\left|\zeta_{1} \zeta_{2}\right|^{(m-1) N-1}\right)\left|\zeta_{1} \zeta_{2}\right|^{N}\|\chi\|_{N-1, p} .
\end{aligned}
$$

From this and from (12), we derive that

$$
\begin{aligned}
\|R(\psi+\chi)-R \psi\|_{N-1, p} & \leq\left\|R_{\operatorname{lin}} \psi\right\|_{N-1, p}+\|Q(\psi+\chi)-Q \psi\| \\
& \leq \frac{1}{3}\|\psi\|_{N-1, p}+K^{\prime}\|\chi\|_{N-1, p} \leq \frac{2}{3}\|\chi\|_{N-1, p}
\end{aligned}
$$

provided $p$ is small enough. Therefore, for $p$ small and $N$ large, the operator $R$ is a contraction on $B_{N-1, p}$. Hence we get a unique function $\psi_{N}$ solving the nonlinear convolution equation

$$
\zeta_{1} \zeta_{2} \psi_{N}=G^{N}\left(\zeta, \psi_{N}\right), \quad \psi_{N} \in B_{N-1, p}
$$

From the construction of $G^{N}$ it follows that for every $N$ (sufficiently large) the function $\psi_{N}+S_{N}$ satisfies the equation (4), hence the $k$ th Taylor coefficient of $\psi_{N}$ (at 0 ) must satisfy (6) (for $k_{i} \geq N+1$ ), so $T$ defined formally by (5) and (6) converges on $\left\{\left|\zeta_{i}\right| \leq p_{i}\right\}$.

2. Analytic continuation of solutions. Define $S(r)=\{\zeta \in \mathbb{C}:|\zeta| \leq r\}$ $\cap S_{1}$ (see Introduction) and let $p$ be such that the solution $\psi_{N}$ of (13) is holomorphic in the interior of $S^{2}(p)=S(p) \times S(p)$. We shall extend this solution to a unique solution on some complex neighbourhood of $\mathbb{R}_{+}^{2}$. 
Choose $\delta, p_{1} \in \mathbb{R}_{+}, \delta<p_{1}<p$. Define

$$
\begin{aligned}
S_{0} & =S\left(p_{1}\right) \times S(p), \\
S_{+} & =\left\{\zeta \in \mathbb{C}^{2}:\left(\zeta_{1}-p_{1}, \zeta_{2}\right) \in S(\delta) \times S(p) \text { or } \zeta_{1}=p_{1}\right\}, \\
S^{1} & =S_{0} \cup S_{+} .
\end{aligned}
$$

Then $S_{0} \cap S_{+}=\left\{p_{1}\right\} \times S(p)$.

Let $W_{0}$ denote the space of functions on $S^{1}$ which are continuous on $S^{1} \backslash\left(S_{0} \cap S_{+}\right)$and analytic in its interior. Next define $\widetilde{\psi} \in W_{0}$ by setting $\widetilde{\psi}=\psi_{N}$ on $S_{0}$ and $\widetilde{\psi} \equiv 0$ on $S_{+}$. Introduce the space

$$
V_{N-1}(\delta)=\left\{\phi \in C^{0}\left(S_{+}\right) \cap \mathcal{O}\left(\operatorname{int} S_{+}\right): \sup _{\zeta \in S_{+}}\left|\zeta_{2}^{-N+1} \phi(\zeta)\right|<\infty\right\} .
$$

For $\phi \in V_{N-1}(\delta)$ define $\phi_{0} \in W_{0}$ by extending $\phi$ by zero on $S_{0}$. Then

$$
\left(\phi_{0} * \phi_{0}\right)(\zeta)=\int_{C(\zeta)} \phi_{0}(\zeta-\gamma) \phi_{0}(\gamma) d \gamma \equiv 0
$$

where $C(\zeta)=C\left(\zeta_{1}\right) \times C\left(\zeta_{2}\right), C\left(\zeta_{i}\right)$ is a path from 0 to $\zeta_{i}$. Hence also $\phi_{0}^{* m} \equiv 0$ for $m \geq 2$. Clearly, $\widetilde{\psi}^{* m}=\psi_{N}^{* m}$ on $S_{0}$ for all $m$. Therefore $(\widetilde{\psi}+\phi)^{* m}=$ $\widehat{\psi}^{* m}+m \widehat{\psi}^{*(m-1)} * \phi_{0}$.

Consequently, for $G(\zeta, \psi)=G^{N}(\zeta, \psi)$ given by (8) we have

$$
\begin{aligned}
G\left(\zeta, \widetilde{\psi}+\phi_{0}\right) & =G(\zeta, \widetilde{\psi})+\left(B * \phi_{0}\right)(\zeta) \quad \text { where } \\
B(\zeta) & =g_{1}(\zeta)+\sum_{m=2}^{\infty} m\left(g_{m} * \widetilde{\psi}^{*(m-1)}\right)(\zeta) .
\end{aligned}
$$

Thus the equation

$$
\zeta\left(\widetilde{\psi}+\phi_{0}\right)=G\left(\zeta, \widetilde{\psi}+\phi_{0}\right)
$$

gives rise to a linear convolution equation

$$
\phi_{0}=\chi+\frac{1}{\zeta}\left(B * \phi_{0}\right)(\zeta)
$$

for $\phi_{0} \in V_{N-1}(\delta)$, with $\chi(\zeta)=\frac{1}{\zeta} G(\zeta, \widetilde{\psi})-\widetilde{\psi}$.

For $\zeta \in S_{+}$and $\phi \in V_{N-1}(\delta)$ we have

$$
\begin{aligned}
\left|\frac{1}{\zeta}\left(B * \phi_{0}\right)(\zeta)\right| & =\left|\frac{1}{\zeta} \int_{p_{1}}^{\zeta_{1}} \int_{0}^{\zeta_{2}} B\left(\zeta_{1}-\eta_{1}, \zeta_{2}-\eta_{2}\right) \phi\left(\eta_{1}, \eta_{2}\right) d \eta_{1} d \eta_{2}\right| \\
& =\left|\frac{1}{\zeta} \int_{0}^{\zeta_{2}}\left[\int_{0}^{\zeta_{1}-p_{1}} B\left(\zeta_{1}-p_{1}-\gamma_{1}, \zeta_{2}-\eta_{2}\right) \phi\left(\gamma_{1}+p_{1}, \eta_{2}\right) d \gamma_{1}\right] d \eta_{2}\right| \\
& \leq \frac{1}{|\zeta|}\|\phi\|_{N-1}\left|\int_{0}^{\zeta_{2}} \eta_{2}^{N-1}\left[\int_{0}^{\zeta_{1}-p_{1}} B\left(\tau, \zeta_{2}-\eta_{2}\right) d \tau\right] d \eta_{2}\right|
\end{aligned}
$$


with $\|\phi\|_{N-1}=\sup _{\zeta \in S_{+}}\left|\zeta_{2}^{-N+1} \phi(\zeta)\right|$. Now, from the definition of $B$, we see that for $\tau \in S(\delta) \times S(p)$,

$$
|B(\tau)| \leq C\left(1+\sum_{m=2}^{\infty} m\left\|\psi_{N}\right\|_{N-1, p}^{m-1} \frac{\Gamma(N)^{2(m-1)}}{\Gamma((m-1) N)^{2}}\left(\left|\tau_{1}\right| \cdot\left|\tau_{2}\right|\right)^{(m-1) N}\right)<M .
$$

Thus for $\zeta \in S_{+}$(and consequently for $\left|\zeta_{1}\right| \geq p_{1}$ ) we have

$$
\left|\frac{1}{\zeta}\left(B * \phi_{0}\right)(\zeta)\right| \leq K\|\phi\|_{N-1}\left|\zeta_{2}^{N-1}\right| \quad \text { with } K=\frac{M \delta}{N p_{1}} .
$$

Hence if we take $\delta<N p_{1} / M$, then the operator $\phi \rightarrow \frac{1}{\zeta} B * \phi_{0}$ is a contraction in the space $V_{N-1}(\delta)$. Thus there exists a unique solution $\phi \in V_{N-1}(\delta)$ satisfying (14). Hence $\phi=\psi_{N}$ on the interior of $S^{2}(p) \cap S_{+}$and it is clear that $\phi$ extends $\psi_{N}$ to $S_{+}$.

A repeated application of this procedure yields an extension of $\psi_{N}$ to some region $U \times S(p)$, where $U$ is a sectorial neighbourhood of $\mathbb{R}_{+}$in $\mathbb{C}$. By interchanging variables and proceeding by the same method we get an extension of $\psi_{N}$ to some region $S(p) \times V$ with $V$ being a sectorial neighbourhood of $\mathbb{R}_{+}$in $\mathbb{C}$. Finally, in the same way we obtain an extension of $\psi_{N}$ to some sector $U \times V$.

3. Exponential estimation. It follows from the results on analytic continuation of the solution of (13) that there exists a function $\psi$, holomorphic in some sector $S$ containing $\mathbb{R}_{+}^{2}$, satisfying (13) and such that $\zeta^{-N+1} \psi$ is locally bounded. We shall prove a global exponential estimate: for every closed subsector $S^{\prime} \subset S$,

$$
\left|\psi\left(\zeta_{1}, \zeta_{2}\right)\right| \leq K\left|\zeta^{N-1}\right| e^{c_{1}\left|\zeta_{1}\right|+c_{2}\left|\zeta_{2}\right|}
$$

for $\zeta \in S^{\prime}$, with appropriate constants $K$ and $c_{1}, c_{2}$. The proof is again a two-dimensional variant of the reasoning given in $[\mathrm{Br}]$.

For $p>0$ define

$$
M(p)=\sup \left\{\left|\zeta_{1}^{-N+1} \psi\left(\zeta_{1}, \zeta_{2}\right)\right|: 0<\left|\zeta_{1}\right|<1,\left|\zeta_{2}\right|=p, \quad \zeta \in S^{\prime}\right\} .
$$

It follows from the local estimates for $\psi$ that $M(p)$ makes sense for each fixed $p>0$. Then for $0<\left|\zeta_{1}\right|<1,\left|\zeta_{2}\right|=p, \zeta \in S^{\prime}$,

$$
\left|\psi\left(\zeta_{1}, \zeta_{2}\right)\right| \leq M(p)\left|\zeta_{1}^{N-1}\right|
$$

and as in (10),

$$
\left|\psi^{* m}\left(\zeta_{1}, \zeta_{2}\right)\right| \leq M^{* m}(p)\left(\frac{\Gamma(N)^{m}}{\Gamma(m N)}\left|\zeta_{1}^{(m-1) N-1}\right|\right)\left|\zeta_{1}\right|^{N} .
$$

Then, by (9), we find that for any $\widehat{c}_{2}>c_{2}$,

$$
\left|\frac{1}{\zeta}\left(g_{m} * \psi^{* m}\right)(\zeta)\right| \leq K e^{\hat{c}_{2} p} * q^{m} M^{* m}(p)\left|\zeta_{1}\right|^{N}
$$


where $q$ is a sufficiently small constant such that

$$
\left(\frac{\Gamma(N)^{m}}{\Gamma(m N)}\left|\zeta_{1}^{(m-1) N-1}\right|\right)^{1 / m} \leq q \quad \text { for } m \in \mathbb{N}
$$

Therefore we have for $0<\left|\zeta_{1}\right|<1,\left|\zeta_{2}\right|=p, \zeta \in S^{\prime}$,

$$
|\psi(\zeta)|=|R \psi(\zeta)| \leq K\left|\zeta_{1}\right|^{N} e^{\hat{c}_{2} p}+K\left|\zeta_{1}\right|^{N}\left(e^{\hat{c}_{2} p} * \sum_{m=1}^{\infty} q^{m} M^{* m}(p)\right)
$$

and for all $p>0$,

$$
\left|\zeta_{1}^{-N+1} \psi(\zeta)\right| \leq \widetilde{K} e^{\hat{c}_{2} p}+\widetilde{K}\left(e^{\hat{c}_{2} p} * \sum_{m=1}^{\infty} q^{m} M^{* m}(p)\right) .
$$

Denoting the right hand side of (15) by $S M$ we get $M(p) \leq S M(p)$ for $p>0$.

Consider the equation

$$
N(p)=S N(p)
$$

Under the Laplace transformation

$$
v(s)=\mathcal{L} N(s)=\int_{0}^{\infty} e^{-p s} N(p) d p
$$

equation (16) becomes

$$
v(s)=\frac{\widetilde{K}}{s-\widehat{c}_{2}}+\frac{\widetilde{K}}{s-\widehat{c}_{2}} \cdot \sum_{m=1}^{\infty}(q v(s))^{m}=\frac{\widetilde{K}}{s-\widehat{c}_{2}} \cdot \frac{1}{1-q v(s)}
$$

or equivalently

$$
q v^{2}-v+\frac{\widetilde{K}}{s-\widehat{c}_{2}}=0
$$

This equation has a unique solution analytic in $1 / s$ at infinity, of the form

$$
v(s)=\frac{\widetilde{K}}{s}+\sum_{l=1}^{\infty} \frac{b_{l}}{s^{l+1}} \quad \text { for } s \text { large enough }
$$

with coefficients $b_{l} \in \mathbb{R}$. Hence

$$
N(p)=\widetilde{K}+\sum_{l=1}^{\infty} \frac{b_{l}}{l !} p^{l}
$$

is a solution of (16) real-valued for $p>0$ and of exponential growth: $N(p) \leq$ $\widehat{\widetilde{K}} e^{\hat{c}_{2} p}$ with some $\widehat{\widetilde{K}}<\infty$. 
Since $M(0)=0$ and $N(0)=\widetilde{K}>0$, and therefore $M(p) \leq N(p)$, it follows from the definition of $M$ that for $\zeta \in S^{\prime} \cap\left\{|\zeta| \leq 1,\left|\zeta_{2}\right| \geq 1\right\}$,

$$
|\psi(\zeta)| \leq \widehat{\widetilde{K}}\left(\left|\zeta_{1}\right| \cdot\left|\zeta_{2}\right|\right)^{N-1} e^{\hat{c}_{2}\left|\zeta_{2}\right|}
$$

By the same method we get for $\zeta \in S^{\prime} \cap\left\{\left|\zeta_{1}\right| \geq 1,\left|\zeta_{2}\right| \leq 1\right\}$,

$$
|\psi(\zeta)| \leq \widehat{\widetilde{K}}\left(\left|\zeta_{1}\right| \cdot\left|\zeta_{2}\right|\right)^{N-1} e^{\bar{c}_{1}\left|\zeta_{1}\right|}
$$

Now we pass to the global estimate on $S^{\prime}$. By (9) we get for $\bar{c}_{i}>c_{i}$ $(i=1,2)$,

$$
|R \psi(\zeta)| \leq K\left(e^{\bar{c}_{1}\left|\zeta_{1}\right|+\bar{c}_{2}\left|\zeta_{2}\right|}+\frac{1}{\left|\zeta_{1}\right| \cdot\left|\zeta_{2}\right|}\left(e^{\bar{c}_{1}\left|\zeta_{1}\right|+\bar{c}_{2}\left|\zeta_{2}\right|} * \sum_{m=1}^{\infty}|\psi|^{* m}(\zeta)\right)\right) .
$$

Using this for $\left|\zeta_{1}\right| \geq 1,\left|\zeta_{2}\right| \geq 1$, since $\psi=R \psi$, we get

$$
\left|\psi\left(\zeta_{1}, \zeta_{2}\right)\right| \leq \widetilde{K}\left(e^{\langle\bar{c},|\zeta|\rangle}+e^{\langle\bar{c},|\zeta|\rangle} * \sum_{m=1}^{\infty}|\psi|^{* m}(\zeta)\right)
$$

As above, under the two-dimensional Laplace transformation we are led to considering the equation

$$
v\left(s_{1}, s_{2}\right)=\frac{\widetilde{K}}{\left(s_{1}-\overline{c_{1}}\right)\left(s_{2}-\bar{c}_{2}\right)} \cdot \frac{1}{1-v\left(s_{1}, s_{2}\right)}
$$

with $v=\mathcal{L} \psi$. Again we prove that it has a solution $v$ analytic in $\left(1 / s_{1}, 1 / s_{2}\right)$ at infinity, so $\psi$ satisfies the exponential growth condition.

\section{References}

[Br] B. Braaksma, Multisummability of formal power series solutions of nonlinear meromorphic differential equations, Ann. Inst. Fourier (Grenoble) 42 (1992), $517-541$

[Sz-Zie] Z. Szmydt and B. Ziemian, The Mellin Transformation and Fuchsian Type PDEs, Kluwer, 1992.

[Zie1] B. Ziemian, Generalized analytic functions with applications to singular ordinary and partial differential equations, Dissertationes Math. 354 (1996).

[Zie2] - Leray residue formula and asymptotics of solutions to constant coefficient PDEs, Topol. Methods Nonlinear Anal. 3 (1994), 257-293.

Institute of Mathematics

Kraków Pedagogical University

Podchorążych 2

30-084 Kraków, Poland

E-mail: smplis@cyf-kr.edu.pl
Institute of Mathematics Polish Academy of Sciences Śniadeckich 8 00-950 Warszawa, Poland E-mail: ziemian@impan.impan.gov.pl 